\title{
Marketing verde en Pymes comercializadoras y distribuidoras de artesanía Wayúu
}

\section{Green marketing strategies in Wayuu SMEs of handicraft marketing and distribution \\ Nair Cantillo Campo \\ Universidad de la Guajira, Riohacha, Colombia.}

Annherys Paz Marcano

Universidad de La Guajira, Riohacha, Colombia.

José Ojeda Hidalgo ii

Universidad Politécnica de Guanajuato, Guanajuato, México.

Resumen

Objetivo: en este estudio se analizó el marketing verde en las Pymes comercializadoras y distribuidoras de artesanía Wayúu con el fin de propiciar la práctica de estrategias que conlleven a la sensibilización en el cuidado y preservación del ambiente. Método: para la realización de esta investigación se utilizó el paradigma positivista, enfoque cuantitativo, tipo descriptiva, diseño no experimental, transversal de campo en la cual participaron 20 encargados y/o propietarios de pequeñas y medianas empresas de artesanía Wayúu registradas en la Cámara de Comercio del distrito especial turístico y cultural de Riohacha, a quienes se aplicó un cuestionario auto administrado, de 12 ítems, con opciones de respuestas múltiples. Resultados: en los hallazgos se encontró que se están utilizando estrategias de marketing verde en las pymes comercializadoras estudiadas, orientadas al reciclaje y reutilización, procurando desde su gestión preservar el medio ambiente. Discusiones: a pesar de que se aplican algunas de las estrategias de marketing verde, estas aún son incipientes, en los comerciantes, por lo que demanda una sensibilización que propicie la cultura ecológica basada en la sostenibilidad del proceso de producción de la artesanía. Conclusiones: se concluye que las Pymes de artesanía Wayúu, con la implementación de estrategias de marketing verde, estimulan el consumo responsable y motivan a sus colaboradores y otros empresarios hacia una actitud ecológica, motivando un patrón de comportamiento compartido en el entorno donde están localizadas.

Palabras clave: ambiente, consumo responsable, cultura ecológica, marketing verde, pymes, sostenibilidad. Clasificación JEL: M31

\section{Abstract}

Autor de

Correspondencia

ncantilloc@uniguajira.edu.co

Recibido: 08-28-2020

Aceptado: 09-06-2021

Publicación: 23-07-2021

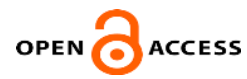

(cc) BY

Copyright (C) 2021

Desarrollo Gerencial
Objective: in this study, green marketing was analyzed in Wayuu handicraft marketers and distributors SMEs in order to promote the practice of strategies that lead to awareness of environmental care and preservation. Method: a positivist paradigm was used, with a quantitative, descriptive, non-experimental, and cross-sectional design. 20 managers and/or owners of small and medium-sized Wayuu handicraft companies participated in the Chamber of Commerce of the special tourist and cultural district from Riohacha, to whom a 12-item selfadministered questionnaire was applied, with multiple response options. Results: it was found that green marketing strategies are being used in the studied marketing SMEs, aimed at recycling and reuse, seeking from their management to preserve the environment. Discussions: although some of the green marketing strategies are applied, these are still incipient, in the merchants, so it demands awareness that fosters an ecological culture based on the sustainability of the craft production process. Conclusions: Wayuu Handicraft SMEs, with the implementation of green marketing strategies, stimulate responsible consumption and motivate their collaborators and other entrepreneurs towards an ecological attitude, generating a pattern of shared behavior in the environment where they are located.

Keywords: environment, responsible consumption, ecological culture, green marketing, SMEs, sustainability. JEL Classification: M31

Como referenciar este artículo (APA):

Cantillo Campo, N., Paz Marcano, A., \& Ojeda Hidalgo, J. (2021). Marketing verde en Pymes comercializadoras y distribuidoras de artesanía Wayúu. Desarrollo Gerencial, 13(1), 1-22. https://doi.org/10.17081/dege.13.1.4408 


\section{Introducción}

La globalización de los mercados ha traído consigo la transformación en la gestión de los negocios, estableciendo acciones para engranar sus procesos de trabajo a los enfoques empresariales vinculados a las tendencias en medios de comunicación, uso de nuevas tecnologías, e innovaciones sociales llevando a evolucionar la vida del hombre e impactada por una precipitada cadena de eventos dinámicos no sólo desde el valor de un ser social por naturaleza sino en el entorno como proveedor de calidad de vida en el espacio donde reside, haciéndole frente a estos factores, y entendiendo que el ser humano es dependiente de la naturaleza y responsable de su preservación y conservación (Lechuga, 2016; Martínez, Cardeño, Ramírez y Durán, 2017).

Dada la severa crisis ecológica que afronta el planeta tierra, la evolución de los esquemas de gestión empresarial requiere plantear acciones destinadas a mitigar los efectos que genera su trabajo en el entorno (Durán, Parra y Márceles, 2015; Ojeda, López y Álvarez, 2019), demandando el fomento de una cultura ecológica responsable en la conservación y preservación de un ambiente sano, ya que entre los factores que colocan en riesgo al ambiente se encuentra la acción humana y empresarial. La evolución de los procesos emanados de ella, conllevan a la aparición de nuevos modelos de producción y comercialización de productos basados en la transformación, uso, consumo excesivo y desmedido de los recursos naturales no renovables, para ser procesados como materia prima, consiguiendo desde su quehacer satisfacer las necesidades que demanda el hombre (López, González, García y Álvarez, 2014).

Las actividades comerciales y de consumo generan efectos contaminantes que impactan la calidad del ambiente debido a los residuos o desechos sólidos que se arrojan y destruyen la diversidad biológica del planeta, afectando el bienestar del ser humano (Choi, 2018) y produciendo el deterioro de la calidad de vida en la tierra. Esto ha llevado a diversos autores, a realizar investigaciones acerca del comportamiento de las empresas en el entorno y a conocer qué tan amigables son las herramientas y técnicas empleadas para desarrollar sus bienes y servicios; lo anterior a expuesto que el gran protagonista del deterioro del ambiente se atribuye a la gestión empresarial y a la poca sensibilidad humana (Cifuentes, Díaz y Osses, 2018; Higuera, Liébana y Muñoz, 2018) pudiendo atribuir a las acciones de disminución de calidad del agua, la desforestación, declive de la biodiversidad; así como el cambio climático ocasionado por la emisión de gases con efectos invernaderos que coloca en riesgo el desarrollo, calidad y bienestar de las personas en su entorno.

Por tanto, a razón de los indicadores de productividad que se establecen como objetivos en las empresas del aparato productivo de las naciones, los líderes mundiales como parte de su gestión, deben asumir un reto ineludible y loable como es la sostenibilidad ambiental (Álvarez y Torres, 2020) trabajando en la defensa del ambiente como uno de los objetivos que aporta desde sus actividades misionales, cerciorándose de que 
Nair Cantillo Campo, Annherys Paz Marcano, José Ojeda Hidalgo

las acciones son parte de una cultura ecológica responsable, reflejada en los resultados alcanzados en las decisiones compartidas en el sector productivo, siendo éstas de naturaleza comercial, consumo, servicios, desarrollo, pesca y agricultura; entre otros; conllevando a garantizar su quehacer en esquemas de gestión verde responsable.

Asimismo, otros sectores del aparato productivo mundial, lo integran las micros, pequeñas y medianas empresas (MiPymes) las cuales han implementado estrategias competitivas efectivas con la dirección de personas que colocan de manifiesto habilidades que promueven un estilo gerencial estratégico para aportar al cuidado y preservación del campo natural con el ambiente, y de manera formal instauran procesos de planificación, organización, dirección y control verde en la gestión de las empresas (Lasarte, Bruna y Duque, 2018; García, Paz y Cantillo, 2019) con el interés por preservar la calidad de vida humana, mediante prácticas corporativas que promuevan el desarrollo sostenible a corto, mediano y largo plazo en respuesta al rescate del ambiente, sin que sus acciones generen una problemática incontrolable que afecte la gestión empresarial.

En tal sentido, esto implica fomentar prácticas ambientalmente responsables en la cadena de valor, desarrollando actividades que contengan la modificación del producto o servicio hacia las tendencias verdes, y que contribuyan a la calidad de vida de la colectividad, favoreciendo los cambios notables en los procesos productivos, en los cuales se ahorren entre los recursos el agua y energía o se produzcan los mínimos desechos. Del mismo modo, insertar acciones verdes, como modificaciones en la distribución, innovaciones tanto en el empaque como en sus actividades promocionales y de publicidad, capacitación de los colaboradores, entre otros aspectos.

De esta manera, el mercadeo ecológico o verde, surge como una actividad estratégica en el quehacer empresarial, según Rodríguez (2011) como mercadeo ecológico o sostenible, se trata de un proceso utilizado en la gestión empresarial para la comercialización de sus productos, mitigando el impacto al ambiente 0 bien la reducción de efectos que su producción genera, mediante una cultura ecológica destinada a la protección y preservación del campo natural, sin crear efectos que perjudiquen al hombre. Es así como, en el campo empresarial, sin exclusión de las pequeñas y medianas empresas de Artesanía Wayúu Colombianas, no se trata de buscar o juzgar los efectos o resultados de su gestión, pues los procesos administrativos son la ventanas que refleja los indicadores de efectividad y eficiencia de la productividad obtenida del desempeño en el entorno, por ello, el marketing, constituye un elemento aliado que vitaliza la imagen y reputación empresarial, correspondiéndole el componente perceptible de la empresa, debido a que su principal propósito es vender una cantidad de productos, y por ende, promover el consumismo, de los productos, bienes o servicios que oferta en el mercado.

De acuerdo con lo anterior, Kotler y Armstrong (2012), sostienen que el marketing es una filosofía de los negocios, clave y esencial en la gestión empresarial, donde su actuación deja ver la práctica de acciones 
Nair Cantillo Campo, Annherys Paz Marcano, José Ojeda Hidalgo

estratégicas ante sus contendientes, a fin de cautivar su mercado meta. Asimismo, estos aspectos coinciden con los aportes Castellano y Urdaneta (2015), en los que coinciden en que el mercadeo verde, representa un conjunto de actividades articuladas en los procesos de gestión empresarial para mitigar los efectos que dañan el ambiente.

Si bien es cierto, las tendencias del mercado global han direccionado a las empresas, sin exclusión de las pequeñas y medianas a la incorporación de términos verdes, entre ellos mercadeo ecológico, como componente en la gestión y filosofía organizacional, dando paso a la reinvención en el desarrollo tanto de los productos como servicios, donde para ello resulta ineludible pensar las necesidades a satisfacer de los consumidores al momento de su elaboración, comercialización y prestación de estos, teniendo en cuenta que estos deben ser cónsonos a los beneficios presentes y futuros de la sociedad, teniendo en cuenta el respeto al entorno natural, social y económico.

Atendiendo a estas consideraciones, Ibarra, Casas, Olivas y Barraza (2015) señalan que el marketing ecológico, consiste en la producción de bienes y servicios sostenibles apegados al criterio de protección y preservación ambiental, con el uso de tecnologías amigables que no atenten contra la naturaleza y el ecosistema, siendo su práctica novedosa en la gestión de negocios, donde se muestra como parte de su estrategia empresarial indicadores de medición de sostenibilidad ambiental, para mantener e incrementar la competitividad y rentabilidad, mejorando al mismo tiempo la imagen corporativa y el cuidado en las comunidad de su entorno. No obstante, Cubillo y Blanco (2014) afirman que el marketing ecológico, debe integrarse en la empresa, desde la filosofía ética de su desempeño social, no solo asumiendo o cumpliendo responsabilidades, sino incorporando esquemas de trabajo sostenibles, donde sus acciones aporten a la protección y cuidado del entorno natural.

Por otra parte, el marketing como estrategia administrativa contribuye al éxito empresarial, con la práctica de mecanismo para posicionar la oferta de sus productos o servicios, logrando el incremento de las ventas así como el consumo de un determinado producto (Lambin, Galluci y Sicurello, 2009; Kotler, 2009; Kotler y Keller, 2012), por tanto, el mercadeo ecológico se centra en el desarrollo sostenible de productos o servicios, ya que orienta a mejorar el crecimiento económico, la equidad social y el valor ecológico en los resultados de los indicadores de gestión de trabajo.

De igual manera, el cambio de intereses, hábitos y actitudes hacia tendencias verdes en el cuidado para la preservación y conservación el entorno natural, donde la educación e información del consumidor son elementos imprescindibles, a los fines de garantizar su imagen y reputación en un mercado cada vez son más exigente. Tal y como afirma Hartman, Forcada y Apaolaza (2004) el marketing verde, está integrado por acciones que orientan el comportamiento del consumidor, entre ellas, informar o educar en argumentos ambientales, incentivando la práctica de actividades favorables a garantizar un ambiente sano, propiciando cambios hacia los valores de la sociedad, donde se estimule e incentive la compra y consumo responsable. 
Por consiguiente, desarrollar productos y empaques ecológicos, se convierte en parte de una cultura ecológica responsable, fortaleciendo la práctica del reciclaje y minimizando el daño en la utilización de materia prima y recursos renovables (Ottman, 2012). Asimismo, según Kotler y Keller (2012) el desarrollo de un producto se debe a diferentes factores estratégicos, al momento de crear un producto, pero cuando se desarrolla en el ámbito ecológico, se debe tener en cuenta los diferentes recursos que la naturaleza presenta.

Por otra parte, la comercialización de productos ecológicos no solo tiene funciones económicas, sino sociales y ambientales, las cuales son valoradas en aspectos tales como la protección de los recursos naturales, beneficios a una comunidad, la comercialización de productos, creación de mercados responsables, entre otras. En este sentido, se plantea los criterios expuestos por Salas (2018) donde señalan que el marketing ecológico adapta las variables del marketing tradicional: Producto, Precio, Plaza y Promoción (4P's); agregándosele otras como la satisfacción y lealtad, teniendo el trabajo como propósito beneficios, tanto para las organizaciones como los clientes.

Dentro de este marco, son diversas las empresas que han abocado sus acciones para desarrollar productos ecológicos a nivel mundial y nacional, desde hace algunos años se ha incrementado la transformación de residuos sólidos en productos rentables, es así como, las empresas en Colombia buscan la incorporación de acciones verdes, donde se propicie esfuerzos en la producción y comercialización de productos ecológicos, destinados a la conservación del medio ambiente, en respuesta a los problemas ecológicos que afronta el planeta, contribuyendo a mitigar efectos que coloquen en riesgos su estabilidad en el mercado, además, desde sus acciones la disminución o eliminación de los daños ambientales derivados sus actividades productivas.

Sin embargo, en el caso del departamento de La Guajira, situado al norte de Colombia bordeado por las agua del Mar caribe, con riquezas naturales centenarias existen dos empresas dedicadas a la preservación y cuidado del medioambiente, siendo estas la Corporación Autónoma de la Guajira (CORPOGUAJIRA) y el Servicio Nacional de Aprendizaje (SENA), las cuales entre sus objetivos esta sensibilizar la conciencia ambiental de la sociedad, mediante la educación y la orientación en la producción y comercialización de productos ecológicos que mitiguen el impacto negativo en la naturaleza.

A pesar de la presencia de instituciones con sentido misional hacia la conservación y preservación ambiental, tanto en empresas como la sociedad en general aún sigue siendo un tema incipiente en el fomento del mercadeo verde con esquemas de trabajo en el desarrollo de productos ecológicos como estrategia amigable con el medioambiente para ser comercializados en la comunidad, y generar cultura de consumo de los mismo e identificándose con la problemática ambiental tanto a nivel general (mundial), como a nivel específico (donde vive), en este caso el exceso de desechos. 
En este sentido, las Pymes como parte del engranaje productivo de este departamento presentan deficiencias con las estrategias de mercadeo ecológico, en específico con la educación ambiental ya que no se imparten charlas o talleres en las cuales se estimule y facilite la aceptación de ideas o comportamientos sociales que se consideren beneficiosos para la sociedad desde la generación de sus productos, lo que significa difundir ideas y conductas medioambientales deseables entre los ciudadanos. También se obvia la elaboración de campañas publicitarias donde se dé a conocer la importancia del reciclaje, la reutilización y reducción de materiales tales como el vidrio, plástico, papel o cartón, ya que estas representan elementos que modifican la conducta de compra del consumidor.

De tal manera, que colocan en riesgo su estabilidad en el incumplimiento de precio, plaza, promoción y distribución de los productos debido a que la mayoría de ellas, no realizan estrategias de mercadeo ecológico como el reciclaje o reutilización, pudiendo desde su quehacer aprovechar para convertir dichos residuos en productos ecológicos que favorezcan a la comunidad, reduciendo de esta manera los desechos, aportando desde su actuación en un ambiente sano y sostenible.

Dado lo anterior, este articulo busca propiciar la práctica de estrategias de marketing verde, a fin de crear una cultura ecológica responsable en el quehacer de la gestión de las Pymes objeto de estudio, conllevando a la sensibilización en el cuidado y preservación del medio ambiente, siendo la dinámica de un desempeño ambiental sostenible, transferible a los clientes y los grupos de interés de la sociedad, considerando que la importancia actual de los negocios, es la elaboración de productos ecológicos, mediante estrategias de reciclaje, reutilización, y reducción, teniendo en cuenta las necesidades del consumidor, la estrategia de los productos verdes, como un marco de referencia hacia el desarrollo sostenible. A raíz de esto, surge la investigación, que tuvo como propósito analizar las estrategias de marketing verde en dichas Pymes del departamento de La Guajira.

Finalmente, este documento presenta una introducción donde describe a nivel general el tema del marketing ecológico, verde o ambiental en las pymes, luego se fundamenta teóricamente el marketing verde con sus correspondientes dimensiones, se establece el proceso metodológico y la recolección de los datos en la población observada, luego muestra los resultados obtenidos, la discusión y posteriormente las conclusiones a las que se llega en la investigación.

\section{Fundamentación teórica}

\section{Marketing Verde}

El marketing verde, ha cobrado cada día importancia en la gestión de las empresas, sobre todo en aquellas que buscan ofrecer productos amigables con el ambiente ya sea mediante la modificación de los 
Nair Cantillo Campo, Annherys Paz Marcano, José Ojeda Hidalgo

productos o realizando cambio en los procesos de producción. Para Larios, Hernández y Hernández (2016) el mercadeo verde comprende el modo de ver y llevar a cabo el vínculo interactivo (sociedad-ambiente), propendiendo a la satisfacción de las partes interesadas, para crear valor, sin colocar en riesgo la preservación y conservación del ambiente, pudiendo con los resultados de las actividades empresariales ayudar al desarrollo sostenible de la economía y la sociedad.

Por su parte, Cubillo y Cerviño (2008) afirman que el marketing ecológico o marketing verde, alude a dos puntos de vista, uno el contexto social, representando el conjunto de actividades realizadas por instituciones sin fines de lucro para sensibilizar y crear una cultura ecológica responsable en la colectividad, y diversos grupos de interés, mientras la otra es el escenario empresarial, con la praxis del marketing, como estrategia de comercialización de productos verdes, en el cual las empresas propenden la búsqueda de acciones que les permita satisfacer las necesidades de los consumidores, tanto por el producto ofertado como en el incentivo de la preservación y protección ambiental.

De acuerdo con Rodríguez (2011) el marketing ecológico más que un proceso es una actividad estratégica empresarial, destinada a la comercialización de sus productos que coadyuva a minimizar el impacto en el ambiente o disminuir los posibles daños a consecuencia de la gestión de sus operaciones de producción, distribución o consumo, creando así en la colectividad el interés por la protección y preservación del ambiente. Al respecto, Lambin, Galluci y Sicurello (2009) sostienen que el mercadeo ecológico tiene como el objetivo principal estimular a ambas partes de la cadena de ventas a desarrollar y distribuir productos sanos y limpios para el mercado en donde se sitúa, generando comportamientos amigables que beneficien tanto al entorno como a los consumidores.

\section{Estrategias de marketing verde}

Con relación al término estrategias, Stanton, Etzel y Walker (2007) señalan que se trata de un sistema de actividades lucrativas, que tiene como eje de acción planear, fijar precios, promocionar y distribuir productos, servicios e ideas a favor de la satisfacción de necesidades entre los mercados meta en la consecución del logro de los objetivos de la organización. En este orden de ideas, las estrategias de marketing, por ser factores capaces de determinar el rumbo de las organizaciones, requieren de un análisis meticuloso y detallado, con la finalidad de no omitir el detalle más mínimo en relación a su diseño e implantación. Así mismo, en su proceso de ejecución se debe realizar evaluaciones permanentes que permitan cumplir los objetivos trazados en el tiempo esperado. Al respecto Pedraza, Cantillo y Dueñas (2019) sostienen que, en todos los procesos de la organización, son es indispensables diseñar estrategias que le permitan alcanzar las metas, convertirse en un marco de referencia para la continuidad en el mercado.

Para autores como Schoell y Guiltinan (2003) una estrategia de mercadeo viene hacer un plan estratégico desarrollados por la organización a través de los recursos disponibles para obtener los objetivos de 
Nair Cantillo Campo, Annherys Paz Marcano, José Ojeda Hidalgo

mercadotecnia; ello lleva implícito la selección y análisis de un mercado meta como el centro del esfuerzo comercial, la creación y mantenimiento de una mezcla de mercadotecnia para satisfacer ese mercado. En tanto, para Kotler (2009) es el conjunto de técnicas, métodos y herramientas empleadas en una empresa para obtener los objetivos planificados; además de metas que servirán como insumos en el diagnóstico de los problemas de la empresa; pudiendo con ello crear correctivos que infieran en las ventas, cuando estas correspondan a la base misional de una organización.

Según Cubillo y Cerviño (2008) la estrategia de marketing ecológico corresponde al plan desarrollado por una compañía para conseguir los objetivos destinados a diseñar, promocionar y establecer las políticas de precios, con el cual se va guiar la distribución de productos verdes en el entorno pudiendo a través de este crear y sensibilizar nuevos hábitos de consumos en la colectividad, con la oferta de productos y servicios respetuosos con el entorno natural. Por su parte, Calomarde (2000) establece que la estrategia ecológica de un producto, servicio o marca debe estar propuesto a crear armonía con el ambiente desde la práctica de estrategias organizacionales, por tanto su redacción deberán tener en cuenta las políticas de ventas de la empresa, y los criterios ecológicas mundiales destinados a generar un desarrollo sostenible desde el hacer y quehacer de sus actividades, mitigando con ello consecuencias que coloque en riesgos un ambiente sano y equilibrado.

De tal manera que, de acuerdo con los criterios argumentativos de Calomarde (2000) las estrategias ecológicas, deben ser alineadas al objetivo empresarial, a fin de que sus resultados puedan impactar a la excelencia en la rentabilidad, e imagen y mantener la competitividad; para ello, su primer paso, para crear la estrategia de mercadeo ecológico, es el diseño y desarrollo de productos ecológicos, lo que lleva consigo:

a) Cambiar los esquemas de trabajo en la producción de sus productos, sin perjudiciales el equilibrio del medio ambiente.

b) Utilizar envases biodegradables que no coloquen en riesgo la calidad del medio ambiente.

c) Minimizar el uso de energías fósiles y materias primas dañinos al medio ambiente

d) Aprobación de los costos sociales que genera la producción y el consumo de los productos.

Finalmente, Calomarde (2000), explica que lo expuesto se ve reflejado en la diferenciación del producto, para eso se propone, una serie de estrategias:

a) Rediseño en los productos existente.

b) Elaboración de productos novedosos.

c) Renovación de productos ecológicamente no aceptables

Al respecto, Seoanez y Angulo (1999) refieren que las estrategias del marketing verde deben tener en cuenta las políticas de ventas de la empresa ya que hay temas ecológicos que afectan globalmente al 
Nair Cantillo Campo, Annherys Paz Marcano, José Ojeda Hidalgo

consumidor, igual que la limitación de los recursos naturales, el desarrollo sostenible frente al consumismo, la lucha contra la contaminación y la minimización del impacto por los residuos mediante la reducción del envase, reutilización y el reciclado.

Desde la perspectiva de Cubillo y Cerviño (2008) la estrategia de mercadeo ecológico es un plan de acción que tienen las organizaciones para cumplir con sus objetivos, encaminados a diseñar, distribuir, fijar precios y comunicar productos respetuosos con el medio ambiente. Calomarde (2000), por otro lado, señala la coherencia que debe haber entre la estrategia ecológica y el objetivo general; dichas estrategias deben contemplar temas que afecten globalmente al consumidor. Por su parte, Hernández y López (2012) manifiestan que las estrategias del marketing ecológico deben incluir factores que ayuden a la preservación del medio ambiente como la reducción de la utilización recursos naturales, el reciclaje, reducción, reutilización desarrollo sostenible, entre otros.

Dadas las condiciones que anteceden, en esta investigación se comparte el planteamiento de Calomarde (2000) pues coincide en que define con más precisión las estrategias de mercadeo ecológico, dando a conocer los temas que más afectan a los consumidores. En este sentido, el primer paso para desarrollar este tipo de estrategias y proponer estrategias nuevas es que las empresas deben estar encaminadas a diseñar, distribuir, fijar precios y comunicar productos respetuosos con el medio ambiente. De acuerdo con esto, Moravcikova, Krizanova, Kliestikova y Rypakova (2017) plantean que la aplicación de estrategias de marketing ecológico contribuyen a que las organizaciones puedan mejorar su imagen, reputación, credibilidad y confianza entre los grupos de interés, entre ellos, sus competidores, debido a la sensibilidad y creación de cambios de comportamiento en el consumo de la colectividad, que de manera evolutiva, se ha inclinado a la adquisición de productos ecológicos, propendiendo la fidelización de los clientes.

\section{Reciclaje}

Para Calomarde (2000) el reciclaje es un proceso fisicoquímico o mecánico en el cual se somete a una materia o un producto ya utilizado a un ciclo de tratamiento total o parcial con el propósito de lograr extraer de él, materia prima o generación de un nuevo producto. Este proceso también alude a la elaboración de materias primas obtenida de desechos, incorporándolos a un nuevo ciclo de vida; este se realiza con el propósito de mitigar los efectos generados de la producción empresarial, el cual ha traído consigo agotamiento de recursos naturales, macroeconómico y eliminar los desechos.

Este autor afirma que existen una diversidad de motivos para reciclar, entre los cuales se encuentran: ahorro de recursos, disminución de efectos contaminantes en el ambiente, prolongación de la vida útil de los materiales generando diversos usos, ahorro de energía, deforestación, reducción del $80 \%$ de los espacios ocupados en basura, descuento en la cancelación de impuestos por concepto de recolección de basura y al mismo tiempo se genera tanto empleo como riqueza. De tal modo, como admite, el autor, que 
Nair Cantillo Campo, Annherys Paz Marcano, José Ojeda Hidalgo

la mayor parte de los desechos son reutilizables y reciclables, el problema se encuentra en la cultura ciudadana que al desconocer cómo crear su separación, lo mezcla generando basura.

Con respecto al tema, Polo (2017) refiere que uno de los elementos esenciales de la comercialización de productos verdes se atribuye al reciclaje, el cual crea una sinergia al establecer la conectividad con el uso de la tecnología limpia, propiciando la transformación para la reutilización de los objetos reciclado. En este particular, se infiere que el proceso de reciclaje, debe ser una actividad ineludible en la gestión empresarial, debido a que sus resultados propenden la conservación y protección del medio ambiente, el cual busca la transformación del desecho en material reutilizable, a fin de garantizar la práctica en la generación de otros productos útiles a la sociedad.

\section{Reutilización}

Para Calomarde (2000), reutilizar es la actividad de volver a usar un objeto, prolongando la vida útil del producto desde su adquisición hasta el momento en que se deja de utilizar; de tal manera, que gran parte de los productos pueden tener más de una vida útil, sea reparándolos o bien aplicando la creatividad e ingenio para proporcionarle otro uso igual o similar. Igualmente, para el autor reutilizar también incluye comprar productos de segunda mano, ya que así se extiende la vida útil del producto; además se reduce el consumo de productos nuevos, convirtiéndose en un modo de reducir los residuos y amenorar efectos contaminantes en los procesos de transformación de materia prima.

Por otro lado, Lara (2008) alude que la reutilización puede ser un proceso más complejo que implica uso de la creatividad, pues una vez que el objeto-mercancía ha cumplido con su función primaria, deben darle una segunda función, generalmente se exigirá un rediseño o adecuación de los objetos. Cabe resaltar que este proceso requiere más información y capacitación sobre las formas principios proceso, ventajas y complicaciones que implica la reutilización de los objetos, así como sus empaques tomando como una labor prioritaria que debe desarrollarse de una forma idónea para lograr tener éxito en el nuevo producto. Para concluir, la reutilización se ha convertido en un proceso habitual en las empresas dedicadas a la conservación y protección del medio dándole una segunda vida útil y alargando la vida de cada producto (Amazonas, De Carvalho, De Andrade, 2018).

\section{Reducción}

En cuando a la reducción, Calomarde (2000) menciona que la reducción obedece a la disminución y racionalización del uso de los recursos naturales posibles, considerando la praxis de las 3R propuestas por el ecologismo para el fomento de hábitos de consumo respetuosos con el ambiente, las cuales se organizan de acuerdo a las prioridades: lo más significativo es reducir el uso de recursos, posteriormente reutilizar los productos para prologar la vida útil y, una vez que el producto no se pueda utilizar más, reciclarlo correctamente; de tal modo que en síntesis las 3R son: Reducir, Reutilizar y Reciclar. Por tanto, la expansión 
Nair Cantillo Campo, Annherys Paz Marcano, José Ojeda Hidalgo

de la cultura ecológica agregado en la gestión de las organizaciones se fundamente en los principios, que fecundan la sostenibilidad de sus funciones, reunidas en " $4 R$ " de la sabiduría ecológica: Reparación, Reacondicionamiento, Reutilización y Reciclaje apoyados en el cumplimiento de los indicadores ambientales.

\section{Educación ambiental al consumidor}

La educativa ambiental en las organizaciones, representa un reto constante al momento de planificar estrategias de mercadeo ecológico, teniendo en cuenta una visión sostenible, soportada en la orientación y guía de una cultura ambiental responsable, reflejado en mecanismos de acción para resguardar la calidad del ambiente, para garantizar la vida en beneficio común. Para Calomarde (2000) la educación ambiental, es una acción de orientación a la práctica de valores éticos, los cuales ayudan a crear un tejido social sostenible con la generación de cambios en el comportamiento de los consumidores; por tanto, su objetivo se centra en la formación de ciudadanos sensibles al cuidado del medio ambiente, los problemas asociados al conocimiento, actitudes, motivaciones, compromiso y aptitudes para trabajar en forma individual y colectiva en la solución de los problemas del entorno en el presente, como los generados de la relación cultura- naturaleza y la búsqueda de alternativas por el bienestar colectivo.

Es así, según lo expresa el autor que se debe vincular al consumidor en temáticas que contribuyan al desarrollo de sociedades sostenibles, mediante estrategias que conlleven a concienciar el consumo responsable, para propiciar al bienestar de las personas. Por consiguiente, la educación ambiental se ha convertido en un desafío no solo para los ambientalistas, sino también para las empresas, las comunidades y consumidores para sensibilizar en cambios de actitudes, creencias, comportamientos con respeto a los problemas ambientales.

\section{Método}

\section{Diseño}

En esta investigación se analizaron e interpretaron los resultados señalados por los encuestados, bajo un lenguaje numérico empleando la estadística descriptiva; representando un paradigma positivista y enfoque cuantitativo. De esta forma, el estudio es de tipo descriptivo, en el cual se compilan e interpretan hechos característicos de la variable marketing verde, mediante la dimensión estrategias del marketing verde, con indicadores tales como reciclaje, reutilización, reducción, educación ambiental, aportando datos extraídos de la opinión de los encuestados en el escenario de estudio, usando técnicas, métodos e instrumentos científicos para alcanzar la medición del objetivo planteado. 


\section{Participantes}

La población en este estudio estuvo constituida por 20 representantes o dueños de Pequeñas y Medianas empresas de artesanías Wayúu legalmente registradas en la Cámara de Comercio del distrito especial turístico y cultural de Riohacha, departamento de La Guajira, Colombia, destacando que existe un número mayor, no conocido, pero con estatus de informalidad legal. Por lo que la población seleccionada, se establece como censo poblacional, no requiriendo cálculo de muestra alguna, por ser un número pequeño, finito y accesible.

\section{Instrumentos}

Para este caso, se utilizó como técnica de obtención de información acerca de la variable marketing verde, en la dimensión estrategias del marketing verde, la encuesta, la cual se emplea para los diseños de campo, aplicándose como instrumento un cuestionario auto administrado a los dueños de empresas de artesanía Wayúu, constituido por 12 ítems con alternativas de respuestas múltiples, siendo este validado por el criterio experiencial de 5 profesionales en la temática de estudio, donde estos tuvieron la responsabilidad de revisar y evaluar tanto la pertinencia como la redacción de cada ítems de la dimensión e indicador.

Posteriormente, se calculó la confiabilidad del cuestionario recurriendo a la opinión de una población similar, en este caso MiPymes de artesanía, no estructuradas formalmente para aplicar la prueba piloto, usando la fórmula Alfa Cronbach logrando un nivel de fiabilidad de 93\%; considerándose adecuado para su utilización en la población estudiada. Luego se analizaron los resultados recogidos de la población mediante la aplicación de la estadística descriptiva, con interpretación de frecuencias tanto absolutas como relativas, aunado a medidas de tendencia central como la media aritmética con interpretación de datos promedios.

\section{Procedimientos}

Para la realización de la investigación, esta inició con la postulación de un proyecto, conteniendo los aspectos alusivos a los criterios del método científico, en el cual se esbozó la descripción de la situación problema y formulación del interrogante de estudio, objetivos de investigación, justificación y delimitación del estudio. Consecutivamente, se realizó la consulta y revisión de trabajos de investigación previos, como fase al estudio del arte de la variable marketing ecológico; asimismo el material referencial de fuentes documentales de autores considerando el sistema de variables y su respectiva operacionalización.

Seguidamente, se desarrolló la metodología, argumentándose epistemológicamente de criterios cuantitativos-positivistas, mediante un basamento descriptivo de las características del fenómeno que se estudia; asimismo un diseño no experimental con el análisis de información trasversal de campo, donde se 
Nair Cantillo Campo, Annherys Paz Marcano, José Ojeda Hidalgo

recogieron los datos de la población utilizando técnicas e instrumentos pertinente a la tipología del estudio, siendo posteriormente organizados y tabulados para construir la interpretación derivada de la practica estadística y la discusión contrastando la teoría de los autores consultados con los hallazgo recogidos de la opinión de las personas involucradas en la población.

Se continuo con la elaboración de lineamientos estratégicos para las pymes de estudio, conclusiones, recomendaciones y referencias bibliográficas que argumentan el recorrido conceptual de la investigación planteada, mediante los criterios de los autores consultados. Concluido los aspectos desarrollados en la investigación, se procedió a elaborar el informe final, así como el presente artículo como producto del trabajo investigativo realizado.

\section{Resultados}

En esta sección se exponen los datos arrojados en la aplicación del instrumento de recolección de datos, sobre la variable marketing verde, a la población conformada por 20 Pequeñas y Medianas empresas de Artesanía Wayúu, legalizadas y formalmente registradas en la Cámara de Comercio del Distrito de Riohacha, cuya razón social se orienta en la venta y comercialización de productos de artesanía Wayúu. Los datos se interpretaron según el rango y categoría señalada en el baremo de interpretación de datos promedios (Tabla 1) e ilustrándose los resultados en el Tabla 2, acerca de las estrategias del marketing verde.

Tabla 1. Baremo de interpretación de datos (medias aritméticas)

\begin{tabular}{cc} 
Rango & Categoría \\
\hline $1,00 \geq 1,80$ & Muy baja \\
\hline $1,81 \geq 2,60$ & Baja \\
\hline $2,61 \geq 3,40$ & Moderada \\
\hline $3,41 \geq 4,20$ & Alta \\
\hline $4,21 \geq 5,00$ & Muy alta \\
\hline
\end{tabular}

Fuente: Elaboración propia (2020).

Tabla 2. Estrategias de marketing verde

\begin{tabular}{|c|c|c|c|c|c|c|c|c|c|c|c|c|c|c|}
\hline \multirow{3}{*}{ Indicadores } & \multirow{3}{*}{$\begin{array}{l}\text { Alternativa de } \\
\text { Respuesta } \\
\text { Ítems }\end{array}$} & \multicolumn{2}{|c|}{$\mathrm{S}$} & \multicolumn{2}{|c|}{$\mathrm{CS}$} & \multicolumn{2}{|c|}{ AV } & \multicolumn{2}{|c|}{$\mathrm{CN}$} & \multicolumn{3}{|c|}{$\mathrm{N}$} & \multirow{2}{*}{\multicolumn{2}{|c|}{ Promedios }} \\
\hline & & \multicolumn{11}{|c|}{ Encargados y/o Propietarios } & & \\
\hline & & FA & $\mathrm{FR} \%$ & FA & FR\% & FA & FR\% & $\mathrm{FA}$ & $\mathrm{FR} \%$ & FA & $\mathrm{FR} \%$ & ITEM & IND. & DIM \\
\hline & 1 & 4 & $20 \%$ & 10 & $50 \%$ & 4 & $20 \%$ & 2 & $10 \%$ & 0 & $0 \%$ & 3,80 & & \\
\hline Reciclaje & 2 & 6 & $30 \%$ & 2 & $10 \%$ & 10 & $50 \%$ & 2 & $10 \%$ & 0 & $0 \%$ & 3,60 & 3,7 & 3,7 \\
\hline
\end{tabular}




\begin{tabular}{|c|c|c|c|c|c|c|c|c|c|c|c|c|c|}
\hline & 3 & 2 & $10 \%$ & 12 & $60 \%$ & 4 & $20 \%$ & 2 & $10 \%$ & 0 & $0 \%$ & 3,70 & \\
\hline \multirow{3}{*}{ Reutilización } & 4 & 2 & $10 \%$ & 16 & $80 \%$ & 2 & $10 \%$ & 0 & $0 \%$ & 0 & $0 \%$ & 4,00 & \multirow{3}{*}{3,3} \\
\hline & 5 & 4 & $20 \%$ & 4 & $20 \%$ & 6 & $30 \%$ & 6 & $30 \%$ & 0 & $0 \%$ & 3,30 & \\
\hline & 6 & 0 & $0 \%$ & 2 & $10 \%$ & 10 & $50 \%$ & 6 & $30 \%$ & 2 & $10 \%$ & 2,60 & \\
\hline \multirow{3}{*}{ Reducción } & 7 & 0 & $0 \%$ & 10 & $50 \%$ & 8 & $40 \%$ & 0 & $0 \%$ & 2 & $10 \%$ & 3,30 & \multirow{3}{*}{4,0} \\
\hline & 8 & 8 & $40 \%$ & 4 & $20 \%$ & 8 & $40 \%$ & 0 & $0 \%$ & 0 & $0 \%$ & 4,00 & \\
\hline & 9 & 8 & $40 \%$ & 10 & $50 \%$ & 0 & $0 \%$ & 2 & $10 \%$ & 0 & $0 \%$ & 4,20 & \\
\hline \multirow{3}{*}{$\begin{array}{l}\text { Educación } \\
\text { Ambiental }\end{array}$} & 10 & 12 & $60 \%$ & 6 & $30 \%$ & 2 & $10 \%$ & 0 & $0 \%$ & 0 & $0 \%$ & 4,50 & \multirow{3}{*}{3,9} \\
\hline & 11 & 16 & $80 \%$ & 0 & $0 \%$ & 4 & $20 \%$ & 0 & $0 \%$ & 0 & $0 \%$ & 4,60 & \\
\hline & 12 & 0 & $0 \%$ & 2 & $10 \%$ & 8 & $40 \%$ & 10 & $50 \%$ & 0 & $0 \%$ & 2,60 & \\
\hline
\end{tabular}

La tabla 2, contiene los resultados de la opinión emitida por los sujetos encuestados acerca de las estrategias de marketing verde en pequeñas y medianas empresas de Artesanía Wayuu; en este sentido, en el indicador reciclaje en el ítem 1 , el $70 \%$ de las personas mencionó que siempre o casi siempre usa métodos de reciclaje en la elaboración de sus productos, mientras el $20 \%$ respondió a veces lo hace, y $10 \%$ casi nunca, siendo el promedio del ítem de 3,8 correspondiendo a una categoría de alta presencia de acuerdo al criterio indicado en el baremo.

En relación con el ítem 2, el 40\% de los sujetos expresó que siempre y casi siempre transforman el desecho de sus productos en material reutilizable para elaborar otros productos útiles a la sociedad, mientras un $50 \%$ puntualizo a veces lo realiza, y $10 \%$, casi nunca. El valor promedio del ítem fue de 3,60 situándose en la categoría de alta presencia, de acuerdo con el baremo de interpretación de los datos promedios. En el ítem 3, los encuestados (70\%) manifestaron que siempre y casi siempre ahorra material al utilizar las técnicas de reciclaje en sus productos, el $20 \%$ respondió que a veces, y $10 \%$ casi nunca lo hace. El promedio del ítem fue de 3,7, que según el baremo de interpretación de datos promedio se reflejó en la categoría de alta presencia.

En este sentido, los resultados emanados en la opinión de las personas encuestadas, en el indicador reutilización, en el ítem 4, el 80\% admitieron que siempre y casi siempre los sujetos evitan contaminar alargando la vida útil del material que usa en su manufactura; seguidamente el $20 \%$ expreso a veces lo evita. El promedio del ítem estuvo en un valor de 4,0 constituyendo una condición de alta presencia. En el interrogante 5 , se logró determinar que un $40 \%$ de los individuos declaró que siempre y casi siempre rediseñan sus productos o les dan una segunda utilidad cuando no son adquiridos. Por otro lado, un $30 \%$ indicó a veces y el otro $30 \%$ opinó que casi nunca. El valor porcentual promedio en la pregunta se registró en la categoría de alta presencia con un índice promedio de 3,30. 
En el reactivo 6, el $10 \%$ de las personas consideraron que siempre y casi siempre han adquirido algún producto de segunda mano para aportar en la disminución de la contaminación ambiental, el otro 50\% respondió a veces, casi nunca un 30\%, y el 10\% consideraron que nunca. El promedio de la pregunta fue de 2,60 estableciendo una categoría moderada. En tanto que el promedio del indicador se sustentó en moderada presencia con un 3,3.

En relación con la pregunta 7 del indicador reducción, el $50 \%$ de los individuos contestaron que casi siempre reduce el consumo de recursos naturales en la elaboración de sus productos, en tanto que el $40 \%$ lo hace a veces y $10 \%$ indicó que nunca. El resultado promedio del reactivo fue de 3,30, situando en la categoría de alta presencia. Posteriormente, en el ítem 8, las personas respondieron, con un 80\%, que siempre y casi siempre disminuye gastos energéticos en su producción, finalmente el $20 \%$ refirió que casi nunca ejecuta tal acción. El valor promedio de la pregunta se situó en 4,00 teniendo una categoría de alta presencia.

En referencia al reactivo 9, un 90\% respondió que siempre y casi siempre aprovechan máquinas y herramientas de bajo consumo para la elaboración de sus productos, el otro $20 \%$ manifestó que casi nunca. El comportamiento promedio del ítem se situó en un 4,20 situándose en la categoría de muy alta presencia, mientras el indicador se anotó en un promedio de 4,0 con categoría de alta presencia.

Siguiendo con la temática, al indagar el indicador educación ambiental, los resultados indican que en la pregunta 10, el $90 \%$ de las personas encuestadas manifestó que siempre y casi siempre se preocupan por formar en sus colaboradores una cultura de educación ambiental, finalmente el $10 \%$ contestó que a veces realiza dicha actividad formativa, el valor prometió del ítem, logró un 4,50 disponiéndose en la categoría de muy alta presencia.

Con respecto al reactivo 11 , el $80 \%$ de las personas expresó que siempre logra motivar a sus colaboradores para que tengan una buena actitud hacia el medio ambiente, el $20 \%$ restante lo hace a veces. El valor promedio comportamental del ítem fue de 4,60 constituyendo una categoría de muy alta presencia. Por otra parte, en la pregunta 12 casi nunca el 50\% de los sujetos aseguró que ha vinculado a sus consumidores en algún tipo de temática de cultura ambiental, por otro lado, el $40 \%$ contestó que a veces, y casi siempre tuvo $10 \%$ de las respuestas. El valor promedio en el comportamiento del ítem fue de 2,60 representando moderada presencia, asimismo el indicador fue 3,9 con un alto nivel de presencia. 
Tabla 3. Dimensión e Indicadores de Estrategia de Marketing Verde

\begin{tabular}{|c|c|c|c|}
\hline Indicadores & Ítems & Indicadores & Dimensión \\
\hline \multirow{3}{*}{ Reciclaje } & 1 & \multirow{3}{*}{3,7} & \multirow{12}{*}{3,7} \\
\hline & 2 & & \\
\hline & 3 & & \\
\hline \multirow{3}{*}{ Reutilización } & 4 & \multirow{3}{*}{3,3} & \\
\hline & 5 & & \\
\hline & 6 & & \\
\hline \multirow{3}{*}{ Reducción } & 7 & \multirow{3}{*}{4,0} & \\
\hline & 8 & & \\
\hline & 9 & & \\
\hline \multirow{3}{*}{ Educación Ambiental } & 10 & \multirow{3}{*}{3,9} & \\
\hline & 11 & & \\
\hline & 12 & & \\
\hline
\end{tabular}

Fuente: Elaboración propia (2020).

\section{Gráfico 1. Dimensión e indicadores estrategia de marketing verde}

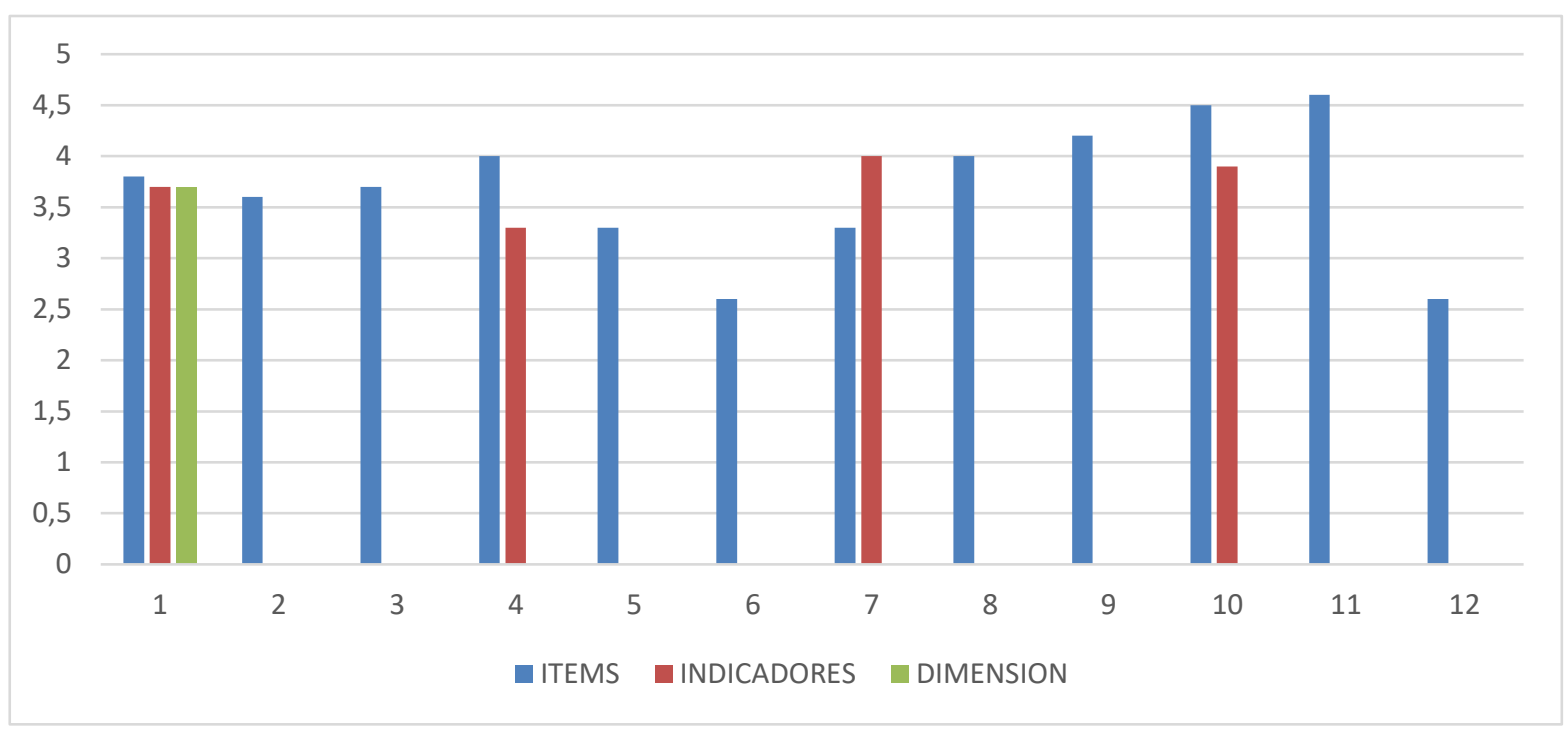

Fuente: Elaboración propia (2020).

Los datos ilustrados en la Tabla 3 y gráfico 1, muestran que el indicador reciclaje, en sus tres ítems obtuvo como resultado 3,7 ubicándose, de acuerdo con los criterios expuestos en el baremo de interpretación de datos promedios, en alta presencia. En tanto, el indicador reutilización arrojó en la sumatoria de la media de sus tres ítems, un valor de 3,3, constituyendo una categoría de moderada presencia. Asimismo, en el indicador reducción, la sumatoria de los tres ítems ofrece un total de 4.0 logrando una categoría de alta presencia. Al hacer referencia al indicador educación ambiental, éste se situó en un valor promedio de 3,9 con alta presencia. De tal forma, que al medir la dimensión estrategia de marketing verde, la misma se ubica con promedio de 3,7, dentro del rango alta presencia en las Pymes estudiadas. 
Con relación a lo expuesto, las Pequeñas y Medianas Empresas de artesanía Wayúu asentadas geográficamente en el distrito especial, turístico y cultural de Riohacha, generan en sus procesos de trabajo ciertos residuos sólidos, tales como, cartón, papel, plástico, vidrio, entre otros, lo que evidencia la falta de compromiso en la gestión de estas empresas hacia la mejora, preservación y conservación y cuidado del medioambiente. Sabiendo que, la mayoría de ellas realizan trabajos productivos en espacios abiertos, propiciando escenarios de riesgo para la sostenibilidad y calidad del medio ambiente, esto quizás por desinformación y desconocimiento de la importancia del mercadeo verde y la elaboración de productos ecológicos para garantizar la distribución y comercialización de excelencia, ganando ventajas competitivas, como resultado de un proceso sustentado en lo ecológico.

En este orden de ideas, se pudo evidenciar que sólo planifican esporádicamente jornadas y recolecciones en sus instalaciones para la venta de los productos ecológicos, iniciativa que de acuerdo al mercadeo se ha tomado con el propósito de incentivar a la sociedad en la compra de dichos productos, pero la mayoría de las veces no ha dado resultados, pues se focalizan en estrategias tradicionales, donde al igual sus productos, quedando rezagada aquellas destinadas a mitigar algún efecto a la sociedad, clientes o consumidores. Asimismo, aseguraron que no se han establecido convenios o alianzas con instituciones ambientales para fomentar el mercadeo y elaboración de productos ecológicos, como aporte a la calidad de vida del hombre.

\section{Discusión}

Teniendo en cuenta el interrogante sobre ¿Cuáles son las estrategias de marketing verde empleadas por las Pymes de Artesanía Wayuu? conlleva a explicar que las transformaciones del mercado global las induce a mirarse desde su interior e identificar la manera en la que se están llevado a cabo los procesos de gestión, para transcender su desempeño y potenciar su posición en contextos económicos, sociales, humanos y ambientales en el mercado donde se desenvuelve, representando esta última dimensión un factor esencial para conservar, preservar y cuidar el medio ambiente, mediante la elaboración y comercialización de productos o servicios amigables con el entorno. De esta manera surge el marketing verde; y por tanto, la implementación de estrategias que contribuyan al desarrollo sostenible, con el consumo responsable, el fomento de la vida de un consumidor más saludable, sin menoscabo de rendimiento económico-financiero.

En ese sentido, los resultados obtenidos en esta investigación muestran que al analizar las estrategias de marketing verde en las Pymes que comercializan y distribuyen artesanía Wayúu, el indicador reciclado presenta un valor promedio 3, 7 ubicándose en la categoría de alta presencia, conforme a los criterios del baremo de interpretación de datos promedios. Este valor reafirma lo planteado por Calomarde (2000), quien asevera que la estrategia del reciclaje es importante por cuanto, al someter una materia o un producto ya utilizado, a un ciclo de tratamiento total o parcial, se logra materia prima o un nuevo producto a partir 
Nair Cantillo Campo, Annherys Paz Marcano, José Ojeda Hidalgo

de desechos; aportando tal acción según Moravcikova, Krizanova, Kliestikova, y Rypakova (2017) y Salas (2018), a la sostenibilidad económica, social y ambiental de las empresas y el país.

En relación con el indicador reutilización, se ubicó en el rango moderado con un valor promedio de 3,3; lo que quiere decir que las pymes en estudio no siempre reutilizan los materiales para la elaboración de sus productos de artesanía, siendo por tanto una estrategia débil, que difiere de la teoría de Calomarde (2000), que considera que al reutilizar se retorna la aplicabilidad de un material u objeto, dándole una segunda vida útil y alargando la posibilidad de uso, pudiendo así generar la cultura de sustentabilidad ambiental en las empresas (Lara, 2008; Ibarra, Casas, Olivas, y Barraza, 2015). En cuanto a la estrategia de reducción, se situó en un valor promedio de 4,0 mostrando alta presencia, coincidiendo dicha estrategia con los criterios de Calomarde (2000), al destacar que la reducción contribuye con la racionalización en el uso de los recursos naturales posibles, considerando la práctica de las tres erres (3R) (Lara, 2008), pudiendo desde las acciones empresariales fomentar hábitos de consumo respetuosos con el medio ambiente (Larios, Hernández y Hernández, 2016).

En lo perteneciente al indicador educación ambiental, los resultados arrojaron un promedio de 3,9 implicando alta presencia en las estrategias de mercadeo verde desarrolladas en las empresas objeto de estudio, siendo esto que coincide con los planteamientos de Hartmann, Forcada y Apaolaza (2004) al exponer que la educación ambiental es responsable de transmitir valores éticos, que contribuyen, al fomento del comportamiento de los consumidores pudiendo desde el desempeño empresarial propiciar la formación de personas hacia el cuidado del medio ambiente tanto de forma individual como colectiva, aportando conocimiento a la solución de los problemas de la sociedad y agregando valor a favor del bienestar y calidad de vida en beneficio de todos.

Con base en lo expresado, se destaca lo significativo de que las Pymes del sector estudiado estén orientadas a generar productos con la filosofía de preservar el ambiente, utilizando estrategias de marketing verde, recomendando afianzar más lo relacionado con la reutilización en la elaboración de las artesanías. Por otra parte, se concluye que se pudo realizar el análisis que da respuesta al objetivo de investigación, sugiriendo líneas de investigación orientadas a las prácticas de reciclaje, reutilización y reducción como estrategias que potencian el marketing verde en las organizaciones.

\section{Conclusiones}

Al analizar las estrategias del marketing verde, se puede evidenciar que las Pequeñas y Medianas empresas de artesanía Wayúu de Riohacha emplean métodos de reciclaje para elaborar sus productos que son comercializados en el mercado nacional e internacional. En este sentido, transforman materiales de desechos, a fin de diseñar artesanía autóctona que satisfaga necesidades a sus clientes; al mismo tiempo que contribuyen con la preservación del ambiente y la cultura del reciclaje para sus generaciones de relevo. 
Por otra parte, en cuanto a la reutilización se observa un comportamiento moderado a pesar, de estar en conocimiento de la importancia de propiciar acciones que mitiguen los efectos que pudiesen crear impacto ambiental en su proceso de elaboración de artesanías desarrollando actividades de rediseño de sus productos logrando expansión de su vida útil y empleando productos de segunda mano a fin de convertirlos en material y con ello, disminuir los indicadores de contaminación al medio ambiente bajo la configuración de acciones alternativas una vez que han sido utilizados.

Asimismo, en la estrategia de reducción, las Pymes objeto de estudio, utilizan material biodegradable para garantizar su proceso de elaboración de artesanías, reduciendo el consumo de recursos naturales, facilitando la promoción de una cultura ecológica entre sus colaboradores, disminuyendo el costo energético durante el proceso de trabajo, empleando herramientas y técnicas amigables con el entorno.

Igualmente, en relación con la educación ambiental, existen indicios de prácticas de acciones para sensibilizar y crear una cultura ecológica basada en la sostenibilidad de su proceso de producción, estimulando y motivando a sus colaboradores hacia una actitud ecológica, con principios y valores ambientales, donde se propicia un patrón de comportamiento compartido con el entorno, en particular los clientes y consumidores, creando ventajas competitivas en ese mercado.

De manera general, el marketing verde en las Pymes estudiada, alude a la práctica de acciones destinadas contribuir con el bienestar y calidad de vida de la sociedad mitigando los efectos que coloque en riesgo el ambiente, por lo que, sus actividades comerciales llevan consigo la elaboración y comercialización de productos amigables al ambiente, sustentando sus procesos desde la vida útil del producto, la elección de materia prima; aunado al retorno del desperdicio para evaluar los criterios de sostenibilidad que beneficien la reutilización, reducción o reciclaje; de tal modo que el enfoque del marketing verde en el quehacer empresarial, no debe estar sujeto a una tendencia moderna sino un modelo de trabajo, que ayuda a disminuir los indicadores negativos que afectan la calidad del entorno natural, involucrando a los grupos de interés tanto internos como externos, entre ellos, los colaboradores y clientes, con el fomento de un consumo responsables, a través de una educación que sensibilice en los problemas y soluciones medioambientales, creando una cultura ecológica que integre a todos en un compromiso compartido.

\section{Financiamiento}

Este artículo es resultado del proyecto de investigación titulado "Marketing Verde como estrategia en el desarrollo de productos ecológicos en la pymes comercializadoras y distribuidoras de artesanía Wayúu", ejecutado entre Diciembre de 2018 - Diciembre de 2019 y financiado por la Universidad de La Guajira. 


\section{Agradecimientos}

Los investigadores expresan su agradecimiento a las personas encargadas de la administración de las Pymes de artesanía Wayúu del distrito especial turístico y cultural de Riohacha, departamento de La Guajira, Colombia, por su contribución al desarrollo de este estudio.

\section{Referencias}

Álvarez, L. \& Torres, A. (2020). Emerging trends in environmental sustainability, telecommunications, industry, and mobility. RIST -Revista Ibérica de Sistemas e Tecnologías de Informacao, (E30), xixii. https://pure.ups.edu.ec/en/publications/emerging-trends-in-environmental-sustainabilitytelecommunication

Amazonas, I., De Carvalho, R. \& De Andrade, M. (2018). Environmental management in hotels: Sustainable technologies and practices applied in hotels. Ambiente e Sociedades, 21, e01722. https://doi.org/10.1590/1809-4422asoc0172r2vu18L1AO

Calomarde, J. (2000). Marketing ecológico. Madrid: Editorial Pirámide- ESIC.

Castellano, S., Urdaneta G. y Joheni A. (2015) Estrategias de mercadeo verde utilizadas por empresas a nivel mundial. Telos, 17(3), 476-494. http://ojs.urbe.edu/index.php/telos/article/view/2265

Cifuentes, F., Díaz, R. \& Osses, B. (2018). Ecology of human behavior: Contradictions behind the message of environmental crisis. Acta Bioethica, 24(2), 161-165. https://dx.doi.org/10.4067/S1726$569 \times 2018000200161$

Choi, J. (2018). Structural relationships among environmental engagement, sustainable behavior, and happiness in university students. Opcion, 34 (Special Issue 15), 924-940. https://produccioncientificaluz.org/index.php/opcion/article/view/30204

Cubillo, J. y Cerviño, J. (2008). Marketing sectorial. Editorial- ESIC.

Cubillo, J. y Blanco A. (2014). Estrategias de marketing sectorial. Editorial-ESIC.

Durán, S., Parra, M. y Márceles, V. (2015). Potenciación de habilidades para el desarrollo de emprendedores exitosos en el contexto universitario. Revista Opción, 31(77), 200-215. https://produccioncientificaluz.org/index.php/opcion/article/view/20052

García Guiliany, J., Paz Marcano, A. y Cantillo Campo, N. (2019). Estrategia y habilidades para la competitividad: caso de pymes del sector construcción en Barranquilla. Aglala, 10(1), 312-339. https://doi.org/10.22519/22157360.1349 
Hartmann, P., Forcada, F. y Apaolaza, V. (2004). Superando los límites medioambientales de la empresa: Un estudio experimental del efecto del posicionamiento ecológico en la actitud hacia la marca. Revista Cuadernos de Gestión, 4(1), 83-95. https://ojs.ehu.eus/index.php/CG/article/view/19186

Hernández, Y. y López, D. (2012). El marketing ecológico y su integración en la planificación estratégica. Venezuela. TELOS. Revista de Estudios Interdisciplinarios en Ciencias Sociales, 14(2), 223-231. http://ojs.urbe.edu/index.php/telos/article/view/2009

Higueras, E., Liébana, F. \& Muñoz, F. (2018). ¿How does pro-environmental behaviour influence the adoption of renewable energies? Dyna (Spain), 94(2), 135-150.

Ibarra, L., Casas, E., Olivas, E. y Barraza, K. (2015). El marketing sustentable como estrategia de posicionamiento global en las franquicias mexicanas que operan en la ciudad de Hermosillo, Sonora. Revista Internacional Administración \& Finanzas, 8(1), 93-109.

Kotler, P \& Armstrong, G (2012). Marketing (14º ed.). Editorial Pearson Educación.

Kotler, P. \& Keller K. (2012). Dirección de Marketing (14º ed.). Editorial Pearson educación.

Kotler, P. (2009). Dirección de Marketing. México. Editorial Pearson Educación.

Lambin, J, Galluci, C y Sicurello, C (2009). Dirección de Marketing. Editorial McGraw-Hill Interamericana Editores.

Lara, J. (2008). Reducir, reutilizar, reciclar. Revista Elementos. Ciencia y Cultura, 15(69), 45-48. https://www.redalyc.org/pdf/294/29406907.pdf

Larios, E., Hernández, E. y Hernández, E. (2016). Comportamiento de consumo y marketing sustentable en México. Brasil. CPMark-Caderno Profissional de Marketing-UNIMEP, 4(2), 18-37. https://www.cadernomarketingunimep.com.br/ojs/index.php/cadprofmkt/article/view/65/69

Lasarte, M., Bruna, D. \& Duque, C. (2018). Tourism planning, promotion and environmental sustainability: The case of Spain. Retos, 8(15), 7-17. https://dx.doi.org/10.17163/ret.n15.2018.01

Lechuga, J. (2016). Editorial. Revista Desarrollo Gerencial, 8(2), 1-13. https://doi.org/10.17081/dege.8.2.2547

López, M., González, E., García, M. y Álvarez, P. (2014). Empirical evidence of the influence of environmental concern on the ecological behaviour of the consumer. Psyecology, 5(1), 58-90. https://doi.org/10.1080/21711976.2014.881666 
Martínez-Ventura, J., Cardeño- Portela, E., Ramírez-Cardeño, W. y Durán, S. (2017). Liderazgo Transformacional como estrategia de adaptación en la gestión logística empresarial. Desarrollo Gerencia/9(2), 140-157. https://doi.org/10.17081/dege.9.2.2980

Moravcikova, D., Krizanova, A., Kliestikova, J. \& Rypakova, M. (2017). Green Marketing as the Source of the Competitive Advantage of the Business. Sustainability, 9(12), 1-13. https://doi.org/10.3390/su9122218

Ojeda-Hidalgo, J., López-Salazar, A., \& Álvarez-Orozco, D. (2019). Does Social Responsibility Influence Organizational Performance? Estudios de Administración, 26(1), 16-34. http://dx.doi.org/10.5354/0719-0816.2019.55404

Ottman, J. A. (2012). As Novas Regras do Marketing Verde: estratégias, ferramentas e inspiração para o branding sustentável. M. Boos do Brasil Editora Ltda.

Pedraza, C., Cantillo, N. y Dueñas, J. (2019). Emprendimiento Social en el sector lácteo. Editorial de la Universidad de La Guajira.

Polo, M. (2017). El Reciclaje: Una alternativa ecológica y de marketing verde para aliviar la contaminación ambiental. https://www.insouthmagazine.com/2017/08/08/el-reciclaje-una-alternativa-ecologicay-de-marketing-verde-para-aliviar-la-contaminacion-ambiental/

Rodríguez, I. (2011). Principios y estrategias de marketing. Segunda Edición. Editorial Eureca Media S.R.L.

Salas Canales, H. (2018). Marketing ecológico: La creciente preocupación empresarial por la protección del medio ambiente. Fides et Ratio, 15(15), 151-170. http://fidesetratio.ulasalle.edu.bo/index.php/fidesetratio/article/view/47

Schoell, W. y Guiltinan, J. (2003) Mercadotecnia. Conceptos y prácticas. Editorial Prentice-Hall Hispanoamericana S.A.

Seoánez, M. y Ángulo, I. (1999). Manual de gestión medioambiental de la empresa. Editortial Mundi-Prensa. Stanton, W., Etzel, M. y Walker, B. (2007). Fundamentos de Marketing. Editorial Mc Graw-Hill. 